\title{
Measurement of a Mirror Surface Topography Using 2-frame Phase-shifting Digital Interferometry
}

\author{
Seok Hee Jeon \\ Department of Electronic Engineering, The University of Incheon, Incheon 402-749, Korea \\ Sang Keun Gil* \\ Department of Electronic Engineering, The University of Suwon, Suwon 440-600, Korea
}

(Received April 9, 2009 : revised April 27, 2009 : accepted April 27, 2009)

\begin{abstract}
We propose a digital holographic interference analysis method based on a 2-frame phase-shifting technique for measuring an optical mirror surface. The technique using 2-frame phase-shifting digital interferometry is more efficient than multi-frame phase-shifting techniques because the 2-frame method has the advantage of a reduced number of interferograms, and then takes less time to acquire the wanted topography information from interferograms. In this measurement system, 2-frame phaseshifting digital interferograms are acquired by moving the reference flat mirror surface, which is attached to a piezoelectric transducer, with phase step of 0 or $\pi / 2$ in the reference beam path. The measurements are recorded on a CCD detector. The optical interferometry is designed on the basis of polarization characteristics of a polarizing beam splitter. Therefore the noise from outside turbulence can be decreased. The proposed 2-frame algorithm uses the relative phase difference of the neighbor pixels. The experiment has been carried out on an optical mirror which flatness is less than $\lambda / 4$. The measurement of the optical mirror surface topography using 2-frame phaseshifting interferometry shows that the peak-to-peak value is calculated to be about $0.1779 \mu \mathrm{m}$, the root-mean-square value is about $0.034 \mu \mathrm{m}$. Thus, the proposed method is expected to be used in nondestructive testing of optical components.
\end{abstract}

Keywords : Topography, Phase-shifting interferometry, Digital holography, Non-destructive testing

OCIS codes : (090.2880) Holographic interferometry; (120.4290) Nondestructive testing; (120.5050) Phase measurement; (120.6660) Surface measurement, roughness

\section{INTRODUCTION}

The measurement of the surface topography in precision elements as well as in optical components is very important. Optical interferometry provides a noncontact and non-destructive test and has an advantage of highly precise measurement with low cost[1-3]. A digital holographic interferometric method that uses a charge coupled device(CCD) camera for direct capture of an interferogram or a hologram has been researched due to advances in imaging technology[4, 5]. Digital holographic interferometry is a good method to measure the surface topography of the test object surface, and the use of digital holographic interference analysis has

*Corresponding author: skgil@suwon.ac.kr been increasing in the precise measurement industry $[3,6]$. Among them, phase-shifting interferometry is a simple and useful technique to reconstruct the object phase information, therefore digital holographic techniques by use of phase-shifting interferometry have been studied to measure the complete surface topography information [7-10] and other optical applications[11-14]. This technique has the merits of simple optical set-up and precise measurement irrespective of light intensity variation, but has an error according to the inconstant change of the amount of phase-shifting. This error can be reduced by using a multi-frame phase-shifting method and error compensation $[8,10]$, but it requires long measurement time and the amount of data is large. Therefore, a 2-frame phase-shifting digital holographic interferometry is able to reduce the number of digital interferograms 
compared to multi-frame phase-shifting methods.

In this paper, we propose a technique for measuring optical surface topography by use of digital holographic interference analysis with a 2-frame phase-shifting method, and to confirm the ability of measuring surface topography with only two interferograms. The basic idea is that we use the relative phase difference of the neighbor pixels of the interferogram and can calculate the phase information of the optical test surface from two digital interferograms. In our measurement system, the Fizeautype phase-shifting interferometer is used to make the digital holographic interferometer. This Fizeau-type interferometer can reduce the influence of environment because the test surface and the reference surface are aligned on the same optical path. In section 2, we describe digital holographic interferometry using 2-frame phase-shifting. In section 3, we describe the optical setup for measuring an optical mirror surface and results of the experiment are shown. The experiment is performed on measuring topography of the optical test mirror. Finally, conclusions are briefly summarized in section 4 .

\section{THEORY}

\section{Digital holographic interferometry using a phase- shifting technique}

Fig. 1 shows the optical experiment set-up of a Fizeautype interferometer using the 2-frame phase-shifting technique[2]. Laser light is collimated by spatial filter SF1 and lens L1. The collimated light going through the beam splitter is traversing a reference flat surface and is propagating to a test surface. The reference surface is attached to a PZT(piezoelectric transducer) and

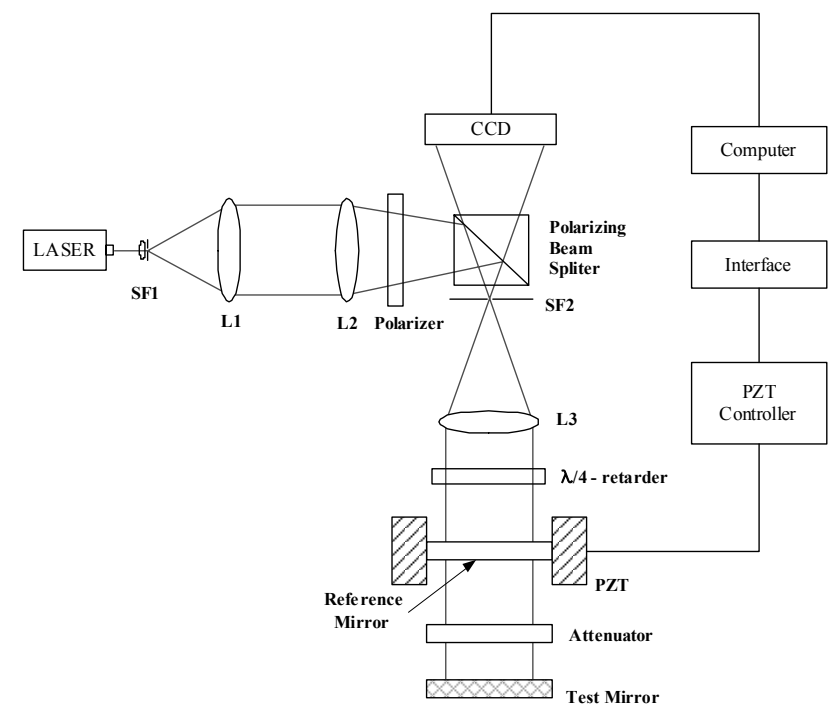

FIG. 1. Optical experiment set-up of Fizeau-type interferometer using 2-frame phase-shifting technique. is moved to allow phase shift by the PZT controller. The beam reflected by the reference surface makes a plane wave as the reference beam and the beam reflected by the test surface makes a wave with phase information as the object beam. These two beams are partially reflected by the beam splitter and are superimposed on the CCD detector by imaging lens L3 to make an interference fringe pattern.

If we let $\overrightarrow{E_{r}}(x, y)=E_{r} \exp \left[j \varphi_{r}(x, y)\right]$ be the reference wave reflected from the reference surface and $\vec{E}_{t}(x, y)=E_{t}$ exp ${ }^{,}\left[j \varphi_{t}(x, y)\right]$ be the object wave reflected from the test surface, the fringe intensity recorded by CCD detector is given by

$$
\begin{aligned}
I & =\left|\overrightarrow{E_{r}}(x, y)+\overrightarrow{E_{t}}(x, y)\right|^{2}=\left(\overrightarrow{E_{r}}+\overrightarrow{E_{t}}\right)\left(\overrightarrow{E_{r}}+\overrightarrow{E_{t}}\right)^{*} \\
& =\left|E_{r}\right|^{2}+\left|E_{t}\right|^{2}+2 E_{r} E_{t} \cos \left(\varphi_{r}-\varphi_{t}\right),
\end{aligned}
$$

where $\varphi_{r}-\varphi_{t}$ is the phase difference between the object and the reference beams, which is proportional to the optical path difference between the reference surface and the test surface, $h(x, y)$. Because $\varphi_{r}$ is constant due to the plane wave reflected from the reference flat surface, the phase information function $\varphi_{t}(x, y)$ of the test surface in terms of the optical path difference $h(x, y)$ is expressed as

$$
\varphi_{t}(x, y)=2 k h(x, y)=\frac{4 \pi}{\lambda} h(x, y),
$$

where $k$ is the wave number and $\lambda$ is the wavelength of the laser light. Therefore, the function $h(x, y)$ represents the test surface topography and can be obtained by

$$
h(x, y)=\frac{\lambda}{4 \pi} \varphi_{t}(x, y)
$$

The phase-shifting interferometer is able to calculate the phase information by changing the relative phase difference between the reference beam and the object beam. Whenever the phase shifting is done, the CCD detector records the interference fringes and stores them in the computer. After multiple interference fringe patterns are acquired by multi-frame phase-shifting, the phase value of each pixel in the fringe pattern can be calculated by analyzing these interferograms. A general method to give rise to phase-shifting is to use a PZT which is attached to the reference mirror. This PZT mirror can give the phase changing of the reference beam due to the expansion effect by applying voltage to the piezoelectric material.

The fringe intensity of the interference is generally given by

$$
I(x, y)=I_{o}(x, y)\{1+V(x, y) \cos \varphi(x, y)\},
$$

where $I_{o}(x, y)$ is the average intensity and $V(x, y)$ is the visibility, and $\varphi(x, y)$ is the phase to be measured. When 
we phase-shift the reference beam by controlling the PZT mirror, we get the phase added to the original reference phase by a phase step. If we let the phase step be $\varphi$ i, the fringe intensity of Eq. (4) is modified into

$$
\begin{aligned}
& I_{i}(x, y)=I_{o}(x, y)\left\{1+V(x, y) \cos \varphi(x, y)-\varphi_{i}\right\}, \\
& \varphi_{i}=(i-1) 2 \pi / R \quad \text { for } \quad i=1,2,3, \cdots, R
\end{aligned}
$$

where $R$ represents the number of the acquired interferograms. From Eq. (5), since variables are $I_{o}(x, y), V(x, y)$ and $\varphi(x, y)$, we need at least three interferograms, that is, $\mathrm{i} \geq 3$ to restore the complete phase $\varphi(x, y)$. For example, four intensity patterns in the form of digital interferograms are achieved by 4 -frame phase-shifting holographic interferometry with the reference beam phase shifted by $\varphi_{i}=0, \pi / 2, \pi$, and $3 \pi / 2$ for $\mathrm{i}=1,2,3,4$.

$$
\begin{aligned}
& I_{1}(x, y)=a(x, y)+b(x, y) \cos [\varphi(x, y)], \\
& I_{2}(x, y)=a(x, y)+b(x, y) \cos [\varphi(x, y)+\pi / 2], \\
& I_{3}(x, y)=a(x, y)+b(x, y) \cos [\varphi(x, y)+\pi], \\
& I_{4}(x, y)=a(x, y)+b(x, y) \cos [\varphi(x, y)+3 \pi / 2] .
\end{aligned}
$$

From Eq. (6), the phase difference of object beam and reference beam is calculated as follows.

$$
\varphi(x, y)=\tan ^{-1}\left(\frac{I_{4}(x, y)-I_{2}(x, y)}{I_{1}(x, y)-I_{3}(x, y)}\right)
$$

\section{Interference fringe analysis using the 2-frame phase-shifting technique}

In order to calculate the exact phase value of each pixel from the fringe intensity pattern recorded on the CCD, more than three interferograms are needed. However, if we align the Fizeau-type optical interferometer set-up so as to have a very small recording angle difference between the reference beam and the object beam, the interference pattern recorded on the CCD has a small number of the white and black fringes. This set-up condition makes the relative phase difference between the neighbor pixels in the interferogram useful to reconstruct the original phase difference of the reference beam and the object beam. Now, we suggest a 2 -frame phase-shifting holographic interferometry with the reference beam phase shifted by $\varphi_{i}=0$ and $\pi / 2$ for $i=1$, 2 , respectively. The piezoelectric controlled reference flat surface mirror is able to allow a phase shift with

\begin{tabular}{|l|c|c|}
\hline$I_{1}(i-1, j)$ th pixel & $(i, j)$-th pixel & $(i+1, j)$-th pixel \\
$I_{1}$ & $I_{1}(\mathrm{i}, \mathrm{j})$ & $I_{1}(i+1, j)$ \\
\hline
\end{tabular}

(a)

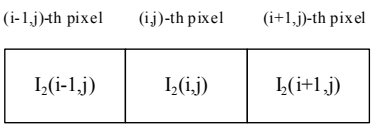

(b)
FIG. 2. Relation between the neighbor pixels in the fringe distribution : (a) when $\varphi_{i}=0$, (b) when $\varphi_{i}=\pi / 2$. phase step of $\pi / 2$ by moving the mirror precisely. This 2 -frame phase-shifting technique is able to reduce the number of interferograms compared to the multi-frame phase-shifting method and to increase the measurement speed in recording the interferograms. By rewriting Eq. (6), two phase-shifting digital interferograms are expressed as

$$
\begin{aligned}
I_{1}(x, y) & =a(x, y)+b(x, y) \cos \varphi(x, y), \\
I_{2}(x, y) & =a(x, y)+b(x, y) \cos [\varphi(x, y)-\pi / 2] \\
& =a(x, y)+b(x, y) \sin \varphi(x, y)
\end{aligned}
$$

where $a(x, y)$ represents the average intensity, meaning the DC-term, $b(x, y)$ represents the modulation intensity, meaning the AC-term, and $\varphi(x, y)$ is the phase to be measured. Fig. 2 shows the relative relation between the neighbor pixels in the fringe distribution.

From the Fig. 2, the corresponding intensity of each pixel is expressed as

$$
\begin{aligned}
& I_{1}(i-1, j)=a(i-1, j)+b(i-1, j) \cos \{\varphi(i-1, j)\}, \\
& I_{1}(i, j)=a(i, j)+b(i, j) \cos \{\varphi(i, j)\}, \\
& I_{1}(i+1, j)=a(i+1, j)+b(i+1, j) \cos \{\varphi(i+1, j)\}, \\
& I_{2}(i-1, j)=a(i-1, j)+b(i-1, j) \cos \{\varphi(i-1, j)+\pi / 2\}, \\
& I_{2}(i, j)=a(i, j)+b(i, j) \cos \{\varphi(i, j)+\pi / 2\}, \\
& I_{2}(i+1, j)=a(i+1, j)+b(i+1, j) \cos \{\varphi(i+1, j)+\pi / 2\},
\end{aligned}
$$

where the $(i-1, j)$-th pixel and $(i+1, j)$-th pixel means the neighbor pixel before and next to the $(i, j)$-th pixel on the horizontal $\mathrm{x}$ direction in the interferogram. Now, the interference pattern is assumed to have a small number of fringes. Since the intensity variation between $(i-1, j),(i, j)$ and $(i+1, j)$ pixel is relatively small compared to the phase variation, and the phase deviations of $(i-1, j)$-th pixel and $(i+1, j)$-th pixel are very small and are approximately similar quantities which have values opposite to each other considering the center $(i, j)$-th pixel phase $\varphi(i, j)$ between them, we can approximate Eq. (9) by assuming $a(i-1, j) \approx a(i+1, j) \approx a(i, j)$, $b(i-1, j) \approx b(i+1, j) \approx b(i, j)$, and $\varphi(i-1, j)=\varphi(i, j)-\theta$, $\varphi(i+1, j)=\varphi(i, j)+\theta$, where $\theta$ is the approximated phase difference between the neighbor pixels.

$$
\begin{aligned}
& I_{1}(i-1, j) \cong a(i, j)+b(i, j) \cos \{\varphi(i, j)-\theta\}, \\
& I_{1}(i, j)=a(i, j)+b(i, j) \cos \{\varphi(i, j)\}, \\
& I_{1}(i+1, j) \cong a(i, j)+b(i, j) \cos \{\varphi(i, j)+\theta\}, \\
& I_{2}(i-1, j) \cong a(i, j)-b(i, j) \sin \{\varphi(i, j)-\theta\}, \\
& I_{2}(i, j)=a(i, j)-b(i, j) \sin \{\varphi(i, j)\}, \\
& I_{2}(i+1, j) \cong a(i, j)-b(i, j) \sin \{\varphi(i, j)+\theta\} .
\end{aligned}
$$

If we let the modified intensities $I_{3}$ and $I_{4}$ be 


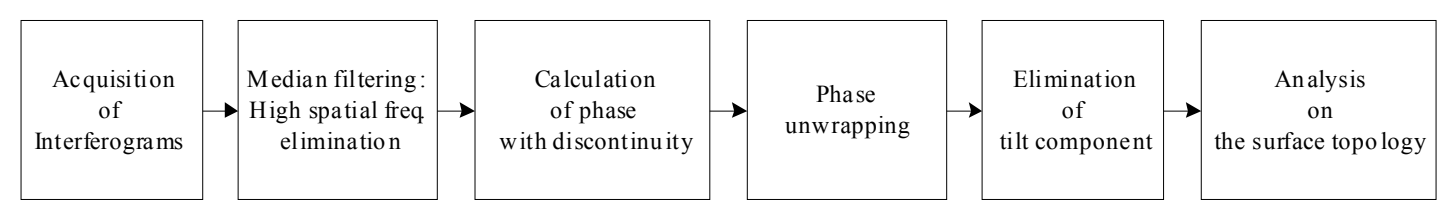

FIG. 3. The procedure of the optical surface topography measurement.

$$
\begin{aligned}
& I_{3}(x, y)=\frac{I_{1}(i-1, j)-I_{1}(i, j)}{I_{2}(i-1, j)-I_{2}(i, j)}=\tan \left\{\varphi(x, y)-\frac{\theta}{2}\right\}, \\
& I_{4}(x, y)=\frac{I_{1}(i+1, j)-I_{1}(i, j)}{I_{2}(i+1, j)-I_{2}(i, j)}=\tan \left\{\varphi(x, y)+\frac{\theta}{2}\right\},
\end{aligned}
$$

then the phase difference of the object beam and the reference beam is calculated as follows.

$$
\varphi(x, y)=\varphi_{r}-\varphi_{t}=\frac{1}{2}\left\{\tan ^{-1} I_{3}(x, y)+\tan ^{-1} I_{4}(x, y)\right\} .
$$

Because the phase value of each pixel in the interferogram is obtained by arctangent calculation using Eq. (12), the phase value exists between $-\pi$ and $\pi$ radian. Therefore, the phase difference $\varphi(x, y)$ between the neighboring pixels can have a discontinuity of $2 m \pi$, where $m$ is an integer. If we let the phase with discontinuity be $\varphi_{d}(x, y)$, this phase must be unwrapped in order to make the phase without discontinuity, $\varphi_{c}(x, y)$, by adding or subtracting the compensating phase $\varphi_{o}(x, y)[8,11]$.

$$
\varphi_{c}(x, y)=\varphi_{d}(x, y)+\varphi_{o}(x, y) .
$$

\section{EXPERIMENT AND RESULTS}

We propose a digital holographic interference analysis method by use of a 2-frame phase-shifting technique for measuring optical mirror surface topography, and show the performance of the proposed measuring technique by experiment. Fig. 1 shows the optical experiment setup for the topography measurement system based on a Fizeau-type phase-shifting interferometer.

This optical surface topography measurement system is made up of the optical set-up, a PZT controller, interface, CCD detector, and data processing computer. A He-Ne laser is used as a light source, the wavelength of which is $0.6328 \mu \mathrm{m}$. Laser light is expanded and collimated by spatial filter SF1 and lens L1. The collimated beam is made into a focused beam by Lens L2 and illuminates the entire polarizing beam splitter(PBS) because the PBS is of relatively small size compared to the diameter of the collimated beam. Since the PBS has the optical characteristics of transmitting p-polarization wave and reflecting s-polarization wave, the polarizer is located and aligned between lens L2 and PBS in order to reflect only the s-polarizing wave of the incidence beam. The beam reflected by the PBS is going through spatial filter SF2 and forms a collimated beam again by lens L3. The purpose of using the spatial filter SF2 is to cut off the beam reflected by lens L3, quarter-wave plate, the reference surface, and the attenuator. The quarter-wave plate is used for changing the incident linear polarized wave into the circular polarized wave and, in reverse, the reflected circular polarized wave into the linear polarized wave. After traversing the quarterwave plate, the s-polarized plane wave is reflected by the reference flat mirror surface and goes back to the quarter-wave plate as the reference beam. The beam traversing the flat reference mirror propagates to a test mirror surface. The wave reflected by the test mirror surface goes back to the quarter-wave plate as the object beam. Since both the reflected reference beam and the object beam are changed into p-polarized waves after traversing the quarter-wave plate, these two beams are transmitted through the PBS and make digital holographic interferograms on the CCD detector. The precisely flat mirror is used as the reference surface attached to the PZT, whose flatness is of less than $\lambda / 20$ and reflectance is about $10 \%$. The back side of this reference flat mirror has an anti-reflection coating to avoid double interference. The transmitted beam intensity through the reference flat mirror is about $90 \%$ of the incidence beam. It is impossible to observe the interference fringe pattern because the intensity of the object beam is 9 times brighter than that of the reference beam. Therefore, the attenuator is inserted in front of the test mirror and is adjusted to make a good interference fringe pattern visibility.

Topography measurement of the test mirror surface is carried out by using the experiment set-up shown in Fig. 1. The test mirror is an optical mirror, model number of which is 02MFG018 (Melles Griot) with flatness of less than $\lambda / 4$. After two interferograms are recorded on the $\mathrm{CCD}$, we carry out a signal processing by computer in order to eliminate high spatial frequency components due to the scattering noise from the test surface. This removal method is done by $3 \times 3$ median filtering process [16]. Fig. 3 shows the procedure in order to calculate the optical surface topography from the acquired interferograms.

Fig. 4 shows two interferograms acquired after median filtering of an interferogram, which is recorded on the CCD detector by using 2-frame phase-shifting digital holographic interferometry. We recognize a little downward movement of the fringe pattern shown Fig. 4, which 


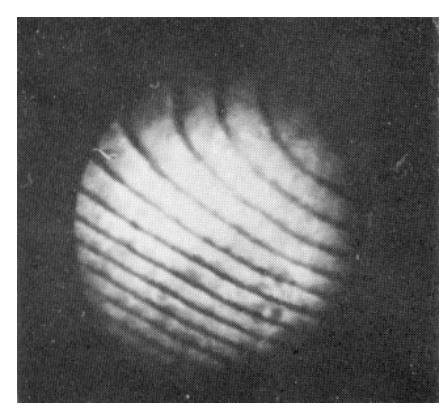

(a)

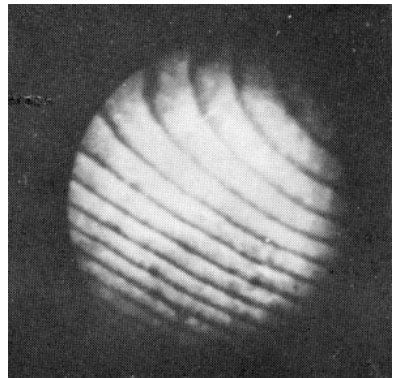

(b)

FIG. 4. Two digital interferograms after high spatial frequency removal : (a) when phase step $\varphi_{i}=0$, (b) when phase step $\varphi_{i}=\pi / 2$.

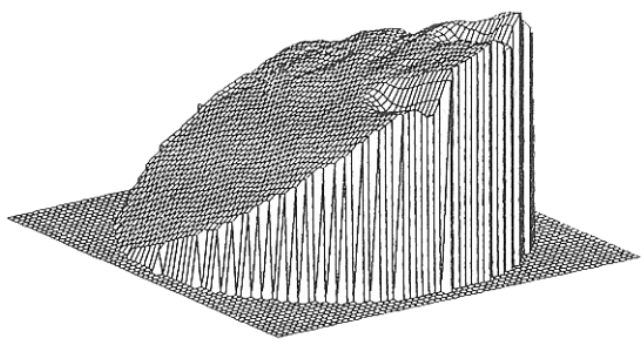

(a)

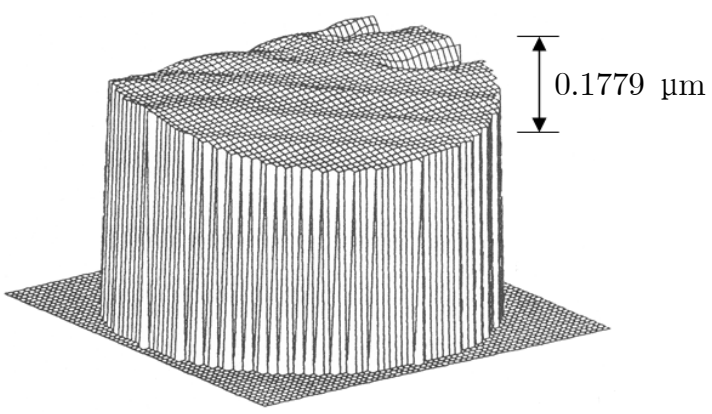

(b)

FIG. 5. The result of the test mirror surface topography measurement using 2-frame phase-shifting interferometry : (a) phase map after unwrapping, (b) the surface topography after eliminating tilt component.

results from the phase-shifting of phase step $\pi / 2$.

From the two interferograms shown in Fig. 4, the phase information is calculated by using Eq. (11) and Eq. (12). Fig. 5 shows the results of the optical mirror surface measurement experiment applying 2-frame phase-shifting digital holographic interferometry. Fig. 5(a) represents the phase map after phase unwrapping, and Fig. 5(b) shows the topography of the test mirror surface after eliminating the tilt component by using Zernike polynomials $[14,15]$. By using Eq. (3), the peakto-peak value is calculated to be about $0.5419 \mu \mathrm{m}$, the root-mean-square value is about $0.157 \mu \mathrm{m}$. However, if we neglect the damaged portion of the optical test mirror on the right, the peak-to-peak value is reduced to about $0.1779 \mu \mathrm{m}$, the root-mean-square value is about 0.034 $\mu \mathrm{m}$. This experimental result is almost within the specification of the test mirror the flatness of which is less than $\lambda / 4(=0.1582 \mu \mathrm{m})$. This means that measurement of the mirror surface topography using 2 -frame phaseshifting digital interferometry is a good method because it takes less calculation time compared to the multi-frame phase-shifting digital interferometric method.

\section{CONCLUSION}

We propose a digital holographic interference analysis technique using a Fizeau-type phase-shifting interferometer for measuring optical surface topography, and a measurement system of the surface topography using 2-frame phase-shifting technique is designed and tested. In the conventional surface topography or flatness test system using phase-shifting interferometry, more than three interferograms are needed at minimum. However, we propose a 2 -frame phase-shifting algorithm which only uses two interferograms. The technique using 2frame phase-shifting digital holographic interferometry is more efficient than multi-frame phase-shifting techniques because the 2-frame method allows less data than multi-frame method for data acquisition and has an advantage of data processing time. The proposed 2-frame phase-shifting algorithm uses the relative phase difference of the neighbor pixels. 2-frame phase-shifting is implemented by controlling the PZT mirror with phase step of 0 or $\pi / 2$ in the reference beam path. Since the optical interferometry is designed on the basis of polarization characteristics of the polarizing beam splitter, the noise from outside turbulence can be reduced. Experiments have been carried out on the optical mirror test. The result of the optical mirror surface topography measurement experiment using the 2-frame phase-shifting technique is very similar to that of the 4-frame phaseshifting method. From the analysis on the surface topography, the peak-to-peak value is calculated to be about 
$0.5419 \mu \mathrm{m}$, the root-mean-square value is about 0.157 $\mu \mathrm{m}$. However, if we analyze interferogram data with smaller size neglecting the boundary damaged portion, the peak-to-peak value is about $0.1779 \mu \mathrm{m}$, the root-meansquare value is about $0.034 \mu \mathrm{m}$. This recalculated result is almost within the specification of the test mirror whose flatness is less than $\lambda / 4$. Thus, the proposed method is able to increase real-time processing and is good for the optical surface topography test, and it is expected to be used in nondestructive testing of optical components and instruments.

\section{ACKNOWLEDGMENT}

This work was supported by University of Incheon Research Grant in 2007.

\section{REFERENCES}

1. D. Malacara, Optical Shop Testing (John Wiley \& Sons. Inc., New York, USA, 1978).

2. Y. I. Ostrovsky, M. M. Butusov, and G. V. Ostrovskaya, Interferometry by Holography (Springer-Verlag, New York, USA, 1980).

3. J. C. Wyant, "Interferometric optical metrology : basic principles and new systems," Laser Focus 18, 65-71 (1982).

4. U. Schnars and W. Jueptner, "Direct recording of holograms by a CCD target and numerical reconstruction," Appl. Opt. 33, 179-181 (1994).

5. B. Skarman, J. Becker, and K. Wozniak, "Simultaneous 3-D PIV and temperature measurements using a new CCD-based holographic interferometer," Flow Meas.
Instrum. 7, 1-6 (1996).

6. J. H. Bruning, "Digital wavefront measuring interferometer for testing optical surfaces and lenses," Appl. Opt. 13, 2693-2703 (1974).

7. I. Yamaguchi, O. Inomoto, and J. Kato, "Surface shape measurement by phase shifting digital holography," in Proc. $4^{\text {th }}$ International Workshop on Automatic Processing of Fringe Patterns (Alkademie, Berlin, Germany, 2001), pp. 365-372.

8. P. Hariharan, "Digital phase-shifting interferometry : a simple error compensating phase calculation algorithm," Appl. Opt. 26, 2504-0505 (1987).

9. I. Yamaguchi and T. Zhang, "Phase-shifting digital holography,” Opt. Lett. 22, 610-612 (1998).

10. J. Schwider, "Phase shifting interferometry : reference phase error reduction,” Appl. Opt. 28, 3889-3892 (1989).

11. S. K. Gil, H. J. Byun, H. J. Lee, S. H. Jeon, and J. R. Jeong, "Optical encryption of binary data information with 2-step phase-shifting digital holography," Proc. SPIE 6488, 648835 (2007).

12. M.-O. Jeong, N. Kim, and J.-H. Park, "Elemental image synthesis for integral imaging using phase-shifting digital holography,” J. Opt. Soc. Korea 12, 275-280 (2008).

13. D. Kim and Y. J. Cho, "3-D surface profile measurement using an acousto-optic tunable filter based on spectral phase shifting technique," J. Opt. Soc. Korea 12, 281-287 (2008).

14. J. Y. Wang and D. E. Silva, "Wave-front interpretation with Zernike polynomials,” Appl. Opt. 19, 1510-1518 (1980).

15. D. Malacara, "Wavefront fitting with discrete orthogonal polynomials in a unit radius circle," Opt. Eng. 29, 672-675 (1990).

16. F. Dubois. O. Monnom, C. Yourassowsky, and J. C. Legros, "Border processing in digital holography by extension of the digital hologram and reduction of the higher spatial frequencies," Appl. Opt. 41, 2621-2626 (2002). 